\title{
Glass reflow on 3-dimensional micro-apertures for electrophysiological measurements on-chip
}

\author{
T. Lehnert · D. M. T. Nguyen · L. Baldi • \\ M. A. M. Gijs
}

Received: 6 April 2006/ Accepted: 19 June 2006/Published online: 18 August 2006

(C) Springer-Verlag 2006

\begin{abstract}
We propose a new method to fabricate micro-apertures for on-chip electrophysiological measurements of living cells. Thermal reflow of phosphosilicate glass (PSG) is applied to funnel- or nozzle-type microstructures to generate very smooth surfaces on the finalized chip. Such 3-dimensional microstructures show close similarities to fire-polished glass pipette tips. Immobilized cells fit perfectly to these structures offering a large contact area for sealing between the cell membrane and the oxide surface. A tight cell/chipaperture seal is an important requirement for the present application. We demonstrate the formation of stable gigaseals with Chinese hamster ovary (CHO) cells for both types of microstructures without the need of any post-fabrication surface treatment. By adjusting the PSG reflow parameters, the shape of the apertures can be modified and diameters down to the submicrometer range may be achieved. The application of PSG reflow to MEMS fabrication is an interesting new option to create unconventional microstructures.
\end{abstract}

Keywords Patch-clamp microsystem - Micronozzle · Microfunnel $\cdot$ PSG reflow

T. Lehnert $(\bowtie) \cdot$ D. M. T. Nguyen · M. A. M. Gijs Institute of Microelectronics and Microsystems,

Ecole Polytechnique Fédérale de Lausanne (EPFL),

1015 Lausanne, Switzerland

e-mail: Thomas.Lehnert@epfl.ch

L. Baldi

Integrative Bioscience Institute,

Ecole Polytechnique Fédérale de Lausanne (EPFL),

1015 Lausanne, Switzerland

\section{Introduction}

The metabolism of living cells depends on the regulation of ion exchange via the cell membrane. Transmembrane proteins act as ion-selective pores that may open or close by responding to external or internal stimuli such as temporary membrane potential changes or ligand binding to receptors on the membrane surface. Any dysfunction of the ion exchange mechanism may cause health problems, the so-called channelopathies, ranging form sufferings such as chronic pain to serious pathologies like Parkinson's or Alzheimer's disease (Ashcroft 2000; Willumsen et al. 2003). Along with the growing knowledge on ion channels, over 400 ion channel coding genes have been identified, the interest of the pharmaceutical industry in developing new drugs that act directly on such targets has strongly increased in recent years (Xu et al. 2001; Worley and Main 2002; Bennett and Guthrie 2003; Willumsen et al. 2003).

The patch-clamp technique is an essential electrophysiological tool in pharma-industry and research laboratories to investigate the impact of new drug candidates or various biochemical compounds on the activity of a specific ion channel. One application of particular importance is safety screening against the hERG (human ether-a-go-go-related gene) potassium ion channel expressed in the human heart, i.e. to track possible hazardous side effects of new active compounds on this target as early as possible (Netzer et al. 2001). In the past, several drugs had to be withdrawn because severe hERG-related perturbations of heart rhythm had not been recognized at an earlier stage of the drug development.

The classical patch-clamp technique is based on a manual handling of individual glass micropipettes, 
allowing only very low throughput (10-20 cells/day). A very important requirement is to obtain an intimate and stable contact between the cell membrane and the material surface, resulting in a tight seal with electrical resistances in the giga-ohm range (gigaseal) (Hamill et al. 1981; Sakmann and Neher 1995). The classical technique cannot step up with the requirements of modern industrial drug development strategies relying on high-throughput approaches (Xu et al. 2001; Comley 2003). Chip-based patch-clamp microsystems are well suited to circumvent the low-throughput bottleneck through robust automated operation and parallelization of electrophysiological measurements. Figure 1 shows a schematic representation of our system. A recent discussion of microchip technology in ion channel research has been given in (Sigworth and Klemic 2005). Overviews on current systems and developments have been given by several authors (Comley 2003; Wang and Li 2003; Lehnert and Gijs 2004; Wood et al. 2004).

Gigaseal formation and pharmacological measurements on cells using planar Si micro-apertures (Asmild et al. 2003; Pantoja et al. 2004), as well as glass chips (Bruggemann et al. 2003; Xu et al. 2003; van Stiphout et al. 2005) have been successfully demonstrated. Seal resistances with hard plastic substrates did not exceed the range of a few hundred mega-ohms (Schroeder et al. 2003). Some recent developments aim to introduce the low-cost elastomer poly-dimethylsiloxane (PDMS), an important aspect in view of a widespread

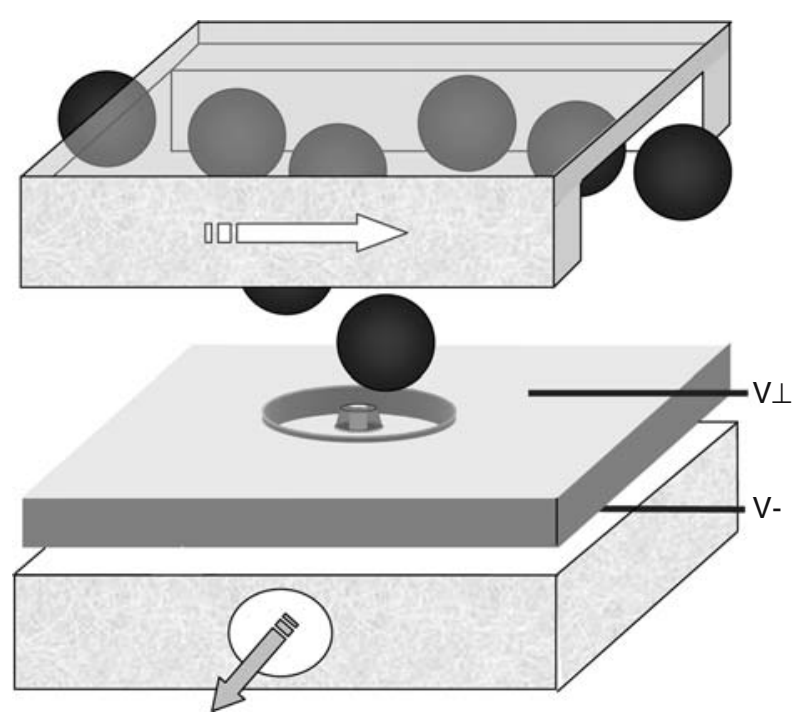

Fig. 1 Schematic representation of the patch-clamp microsystem. Cells are introduced via a fluidic channel (top plate) and positioned on a $\mathrm{Si} / \mathrm{SiO}_{2}$ micro-aperture ( $\mathrm{Si}$ chip in the middle) by suction (base plate). Electrical contacts are on both sides of the aperture utilization of the patch-clamp on-chip technology (Klemic et al. 2002, 2005; Ionescu-Zanetti et al. 2005). Oxygen-plasma treatment of PDMS is normally required to create hydrophilic surfaces. PDMS coating of solid $\mathrm{Si} / \mathrm{SiO}_{2}$ nozzles was tested and the possible formation of gigaseals was observed (Lehnert et al. 2003). Recently, a PDMS-based multi-patch system has been proposed (Ionescu-Zanetti et al. 2005). The authors stated that gigaseals, although with a low yield of about $5 \%$, could be obtained with unmodified, i.e. hydrophobic, PDMS surfaces.

Transposing the classical technique to a chip-based system is not straightforward; especially seal formation with on-chip apertures has been generally recognized as a major problem. This motivates our work to explore routes for more sophisticated (sub)micro-apertures in view of improving the cell-to-aperture seal quality, i.e. yield, stability and resistance. We focus especially on chips that may seal without additional surface modification. We propose in this paper to take advantage of thermal reflow of phosphosilicate glass (PSG) to create 3-dimensional microstructures with very smooth surfaces and optimized shape. The reflow technique is applied to two different microstructures: a new microfabricated $\mathrm{Si} / \mathrm{SiO}_{2}$ funnel structure and the previously developed $\mathrm{SiO}_{2}$ micronozzles (Lehnert et al. 2002). This approach will help to clarify which aperture type and shape is best suited for patch-clamp on-chip experiments.

\section{PSG reflow process}

Reflow of PSG layers by thermal treatment is well known in microelectronic circuit fabrication for gap filling and planarization (Vassiliev et al. 2000). Rapid thermal processing or longer furnace heating in different gas atmospheres has been considered. The typical temperature ranges from 900 to $1,100^{\circ} \mathrm{C}$ (Adams and Capio 1981; Hara et al. 1984). To our knowledge, PSG reflow has not been applied to MEMS fabrication so far.

We introduce PSG reflow to obtain 3-dimensional microstructures with a perfectly smooth surface. Such surfaces, in general, cannot be achieved with Si microstructures fabricated by means of dry etching techniques. Micromasking due to polymer residues results in a non-negligible surface roughness. In our process, a PSG layer was deposited on the whole wafer after processing the microstructures. The PSG was deposited by a low-temperature low-pressure chemical vapor deposition (LPCVD) process. The layer incorporates $6 \mathrm{wt} \%$ of phosphor. A nominal thickness of $1-2 \mu \mathrm{m}$ is 
suitable. Because of its phosphor ion content, the softening temperature of PSG is significantly lower than that for pure amorphous $\mathrm{SiO}_{2}$ layers (approx. $1,600^{\circ} \mathrm{C}$ ) for which laser heating would be required. However, laser treatment of $\mathrm{Si} / \mathrm{SiO}_{2}$ MEMS is not possible. The underlying opaque $\mathrm{Si}$ substrate absorbs more energy and melts faster than the transparent $\mathrm{SiO}_{2}$ coating, resulting in cracking of the solid oxide surface.

In this work, thermal treatments were carried out in a laboratory chamber furnace (Heraeus K114) in ambient air. Quite slow heating and cooling rates apply $(\approx 100 \mathrm{~K} / \mathrm{h})$. The reflow target temperature was maintained for $30 \mathrm{~min}$. Reflow experiments were carried out at $900,1,000$ and $1,100^{\circ} \mathrm{C}$. In order to demonstrate the effect of surface smoothing, a 1.5- $\mu \mathrm{m}$-thick (nominal thickness) PSG layer was deposited on a black-Si surface (Jansen et al. 1995). This test surface was generated by a dry etch process. Its topography consists of a dense distribution of peaks (rms roughness $\mathrm{Rq} \approx 300 \mathrm{~nm}$ ) and exceeds by far the roughness of our as-fabricated microstructures. Nucleation of the LPCVD oxide at the tip of the peaks resulted in a cauliflower-like feature after PSG deposition $(\mathrm{Rq} \approx 250 \mathrm{~nm})$. The atomic force microscope (AFM) picture and profile in Fig. 2a show the PSG surface after annealing at $900^{\circ} \mathrm{C}$. Only very moderate reflow occurred at this temperature. The topography did not change significantly, but smoothing was observed $(\mathrm{Rq} \approx 150 \mathrm{~nm})$. When applied to MEMS fabrication, this temperature is suitable when smoothing of surfaces and rim edges is desired without changing the overall shape of the microstructure. As the initial roughness of the microstructures considered in this paper is much

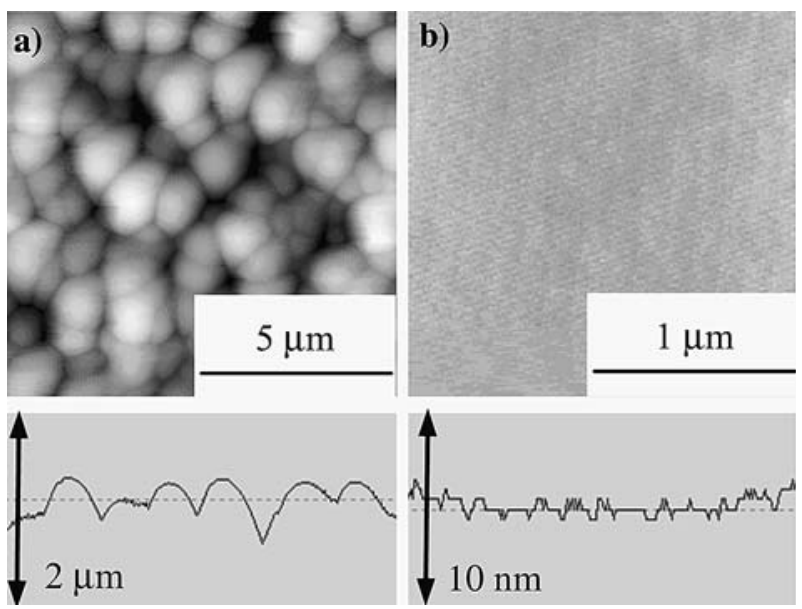

Fig. 2 AFM pictures and scan profiles of a black-Si surface with a LPCVD-deposited PSG layer (1.5- $\mu \mathrm{m}$ nominal thickness). a After reflow at $900^{\circ} \mathrm{C}$ for $30 \mathrm{~min}$, b surface smoothing after reflow at $1,100^{\circ} \mathrm{C}$ for $30 \mathrm{~min}$ smaller compared to the test sample, very efficient smoothing can be expected at $900^{\circ} \mathrm{C}$. At $1,000^{\circ} \mathrm{C}$ further smoothing and clustering are observed with the test sample $(\mathrm{Rq} \approx 100 \mathrm{~nm})$. Figure $2 \mathrm{~b}$ shows a picture and a surface scan after annealing at $1,100^{\circ} \mathrm{C}$. The surface is perfectly flat and the roughness measurement is limited by the noise of the instrument $(\mathrm{Rq}<5 \mathrm{~nm})$. These observations indicate that with a PSG layer applied to a microstructure, not only smoothing but also a change in shape will be introduced after annealing at $1,000^{\circ} \mathrm{C}$ or higher temperatures.

\section{3-Dimensional patch-clamp micro-apertures}

\subsection{Microfunnels}

A Si microfunnel structure was developed by applying different dry etching sequences. The process flow of the main steps is shown in Fig. 3. A microhole with a diameter of $1-2 \mu \mathrm{m}$ in a $0.5-\mu \mathrm{m}$-thick thermal oxide layer on the $\mathrm{Si}$ wafer surface serves as etch mask (Fig. 3a). Then a Si isotropic dry etch process is applied using $\mathrm{SF}_{6}$ as the etchant. The nominal lateral etch rate is $5 \mu \mathrm{m} / \mathrm{min}$. As illustrated in Fig. 3b the strong underetch of the oxide mask due to the selective chemical action of the gas results in a hemisphere-like structure. In the final device a cell will be positioned in this hemisphere. The diameter of the hemisphere is not very sensitive to etch time and to mask hole diameter. Typical values for its diameter at the wafer surface are $6 \mu \mathrm{m}$ after $1 \mathrm{~min}$ of etching and $7 \mu \mathrm{m}$ after $2 \min 30 \mathrm{~s}$ with a $1-\mu \mathrm{m}$ mask hole, or 7 and $9 \mu \mathrm{m}$ for a $2-\mu \mathrm{m}$ mask

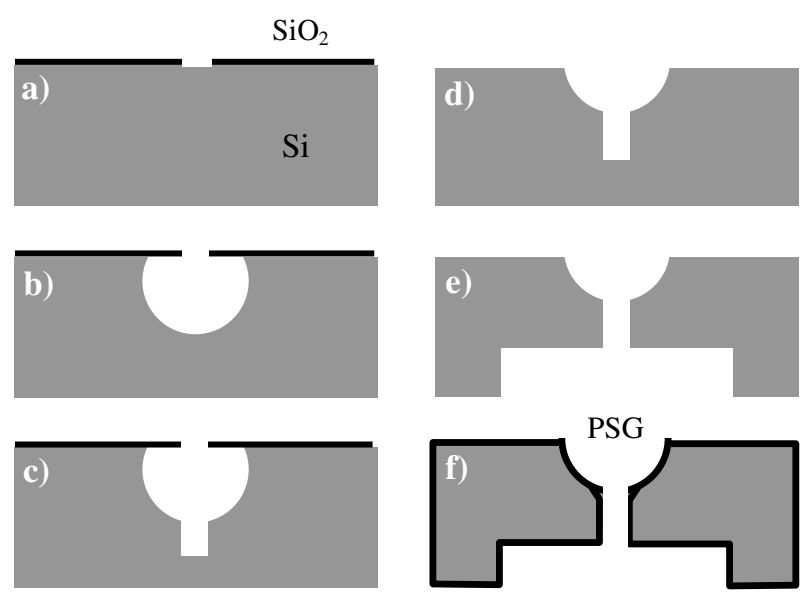

Fig. 3 Microfunnel fabrication process. a Hole in oxide mask $(\varnothing$ 1-2 $\mu \mathrm{m})$, b isotropic Si dry etching, $\mathbf{c}$ DRIE of microhole in $\mathrm{Si}, \mathbf{d}$ CMP on front-side to remove overhang, e backside opening and thermal oxidation, $\mathbf{f}$ oxidation and deposition of PSG 
hole, respectively. Underetching is obviously not a linear process in time in this case. As shown in the schematic view, the hemisphere has generally an overhang of $1-2 \mu \mathrm{m}$. The roughness of the sidewalls of the hemisphere which will be in contact with the cell membrane was minimized by adjusting the etch parameters, but it still features a dense distribution of relatively sharp nanostructures with peak-to-peak values up to about $50 \mathrm{~nm}$. This is not sufficiently smooth to allow gigaseal formation.

Subsequently, the same oxide mask is used to transfer the hole in the mask to the bottom of the hemisphere by a DRIE (deep reactive ion etching) process $\left(\mathrm{C}_{4} \mathrm{~F}_{8}+\mathrm{SF}_{6}\right)$. A small tube-like hole (Fig. 3c) is etched through the bottom of the hemisphere to obtain a link to a larger hole in the backside of the chip. The distance of a few micrometers between oxide mask and microstructure surface results in an enlargement of the diameter of the tube with respect to the hole diameter in the mask. The resulting typical tube diameter is $3.5 \mu \mathrm{m}$ for 1 or $2 \mu \mathrm{m}$ holes in the mask (depth $20 \mu \mathrm{m}$ after 10-min etch time). Hereafter, the oxide mask was removed by BHF wet etch (buffered HF: $\mathrm{NH}_{4} \mathrm{~F}(40 \%): \mathrm{HF}(49 \%)$, ratio $\left.7: 1\right)$.

The slight overhang of the hemisphere would hinder a close contact of the cell with the surface and can damage the cell membrane during immobilization. This overhang is removed by applying a chemicalmechanical polishing (CMP) process to the surface of the wafer for a few minutes (Fig. 3d). The resulting diameter of the hemisphere at the wafer surface is 9$10 \mu \mathrm{m}$ with a depth of 4-5 $\mu \mathrm{m}$. A large backside hole (diameter $500 \mu \mathrm{m}$ ) is then etched by DRIE for fluidic and electrical access to the cell (Fig. 3e). The finalized Si chip is oxidized, followed by the LPCVD deposition of a PSG layer (Fig. 3f).

The as-fabricated structure has two drawbacks for the given application, the non-negligible roughness of the hemisphere and the relatively large tube diameter of about $3.5 \mu \mathrm{m}$ where the small deformable cells (diameter $\approx 15 \mu \mathrm{m}$ ) could be easily sucked through. Preliminary surface smoothing can be achieved by applying a double oxidation process. A $0.5-\mu \mathrm{m}$-thick sacrificial oxide layer is grown on the whole structure by wet oxidation and is subsequently removed by wet etch in BHF solution. Small peaks can be removed by this method, leaving a smoothed Si surface. For good electrical insulation, a second oxidation is carried out, covering the whole structure again with a dense 0.5$\mu$ m-thick $\mathrm{SiO}_{2}$ layer. A scanning electron microscope picture of such a funnel structure (without PSG layer) is shown in Fig. 4a. The resulting oxide surface is smoother than the initial one before the second oxidation. Nevertheless the surface is not smooth enough to allow tight fluidic and electrical seals with a cell membrane.

Figure $4 \mathrm{~b}$ shows a cross section of a microfunnel with an as-deposited PSG layer. The nominal thickness
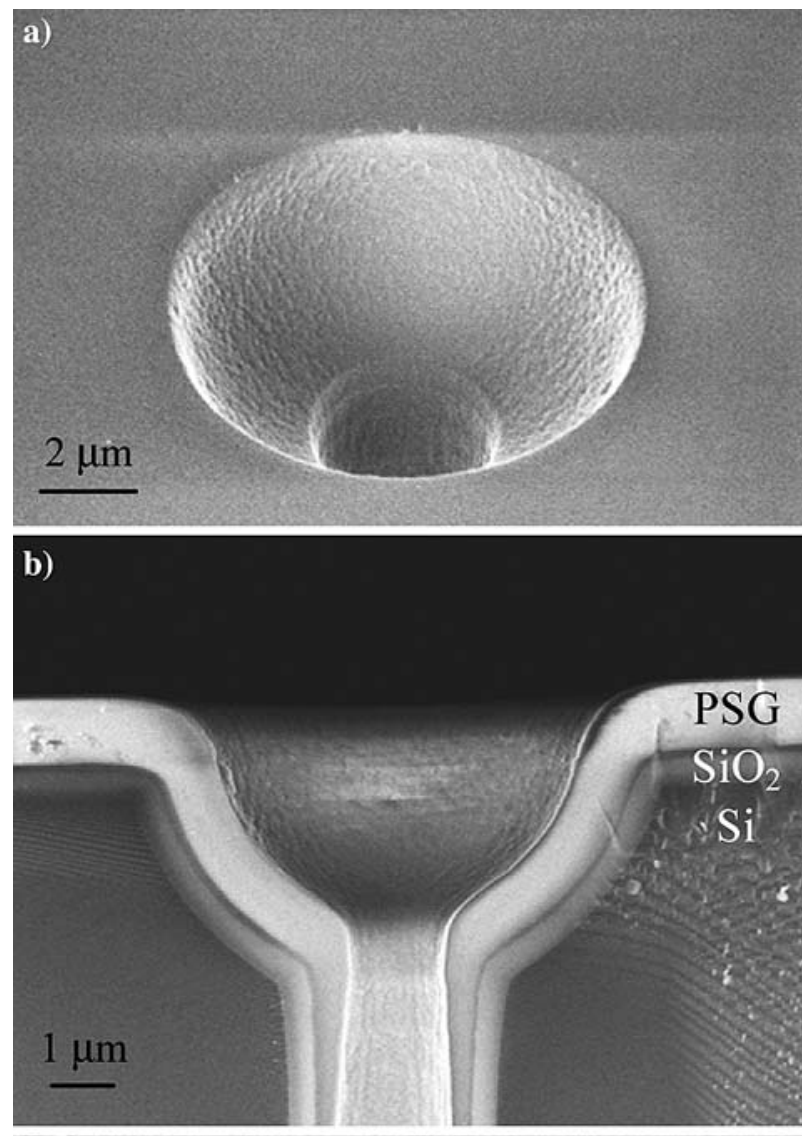

c)

$1 \mu \mathrm{m}$

Fig. 4 a Microfunnel after double thermal oxidation (without PSG). The surface roughness is low but has to be further reduced for good cell sealing. b Cross section of a microfunnel structure with a $1.5-\mu \mathrm{m}$ as-deposited PSG layer on the top of a $0.5-\mu \mathrm{m}$ thick $\mathrm{SiO}_{2}$ oxide layer. No annealing was carried out. c Top view of a very smooth microfunnel after reflow of a $1.5-\mu \mathrm{m}$-thick PSG layer at $900^{\circ} \mathrm{C}$ 
is $1.5 \mu \mathrm{m}$. The thickness is uniform on the horizontal areas of the wafer but decreases rapidly inside the narrow oxide tube. This may be considered as an advantage for the present application because only the diameter of the aperture where the cell will be immobilized has to be minimized. Narrowing the inner tube diameter itself would increase the fluidic and electric access resistance to the cell. Aperture diameters down to $1 \mu \mathrm{m}$ can be readily achieved with an initial tube diameter of about $3 \mu \mathrm{m}$.

Annealing at $900^{\circ} \mathrm{C}$ was applied to a structure similar to Fig. 4b. The resulting microfunnel is shown in Fig. 4c. The overall shape of the funnel structure was not altered. However, significant smoothing of the funnel surface and rounding of the rim edge could be achieved. This microstructure is suitable for patchclamp experiments. Further experiments revealed that at $1,000^{\circ} \mathrm{C}$, the overall funnel shape is still present, although altered by an enhanced reflow. After the reflow at $1,100^{\circ} \mathrm{C}$, the funnel structure was completely plugged and the microstructure was nearly fully planarized (photos not shown).

\subsection{Nozzle-type microstructures}

Previously, we developed $\mathrm{Si} / \mathrm{SiO}_{2}$ micronozzles with the goal to reproduce on-chip the tip of a classical patch pipette (Lehnert et al. 2002). Typical seal resistance values in the range of 50-200 $\mathrm{M} \Omega$ have been achieved. No gigaseals formed with these bare $\mathrm{SiO}_{2}$ micronozzles, most likely because of the non-negligible surface roughness introduced by the DRIE process.

$\mathrm{SiO}_{2}$ CVD deposition on micronozzles and planar apertures has been used previously to reduce the opening diameter, but not for surface smoothing (Luginbuhl et al. 2000; Pantoja et al. 2004). Reflow of pure $\mathrm{SiO}_{2}$ cannot be achieved with normal laboratory equipment (furnaces are limited at best to $1,100^{\circ} \mathrm{C}$ ). Therefore, in order to improve the surface properties of this type of microstructure, we applied the same technique as described in the previous section. A PSG layer was deposited on the final micronozzle wafer followed by thermal treatment for reflow. Deposition and annealing parameters are equal to those indicated before (except layer thickness). The initial bare $\mathrm{SiO}_{2}$ nozzle has a diameter of $3 \mu \mathrm{m}$ and a height of $0.5 \mu \mathrm{m}$. A first annealing experiment at $800^{\circ} \mathrm{C}$ revealed that no significant PSG reflow occurs at this temperature (photo not shown). The surface topography of the PSG on the nozzle rim is comparable to the as-deposited layer. At $900^{\circ} \mathrm{C}$ and for a PSG thickness of $2.0 \mu \mathrm{m}$, moderate reflow was clearly observed (Fig. 5a). The overall shape of the coated nozzle remained unaltered; however, the nozzle rim surface and the inner aperture surface became perfectly smooth. Strong reflow occurred after annealing at 1,000 or $1,100^{\circ} \mathrm{C}$ (PSG thickness $2.0 \mu \mathrm{m}$, Fig. $5 \mathrm{~b}$ ). The nozzle structure disappeared under the PSG layer and

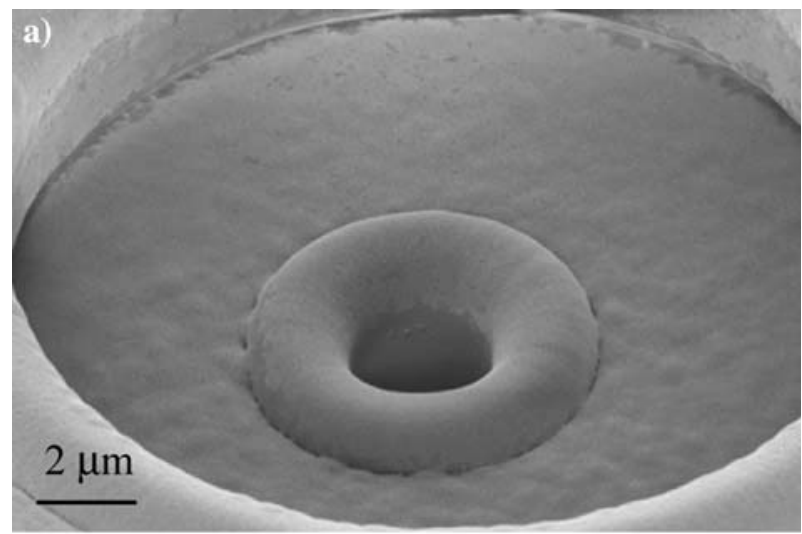

b)

$1 \mu \mathrm{m}$

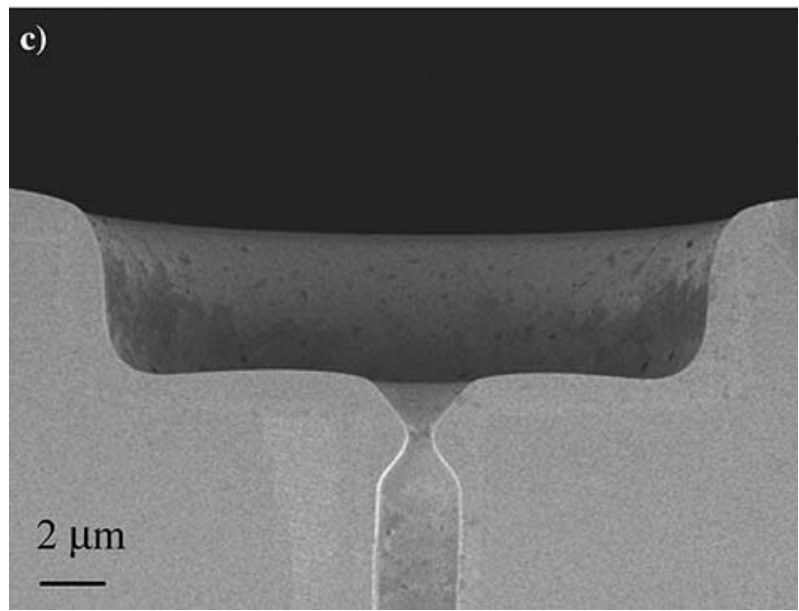

Fig. 5 a, b $\mathrm{SiO}_{2}$ micronozzles after PSG layer deposition (thickness $2.0 \mu \mathrm{m}$ ) and thermal treatment at 900 and $1,100^{\circ} \mathrm{C}$, respectively. Surface smoothing and change in shape can be observed for increasing temperature. c Cross section through a funnel-type microstructure as shown in $\mathbf{b}$ (reflow at $1,100^{\circ} \mathrm{C}, \mathrm{PSG}$ thickness $1.5 \mu \mathrm{m}$ ). A constriction (diameter of $0.9 \mu \mathrm{m}$ ) can be seen at the upper end of the oxide tube. This shape is expected to be very suitable for cell sealing and whole-cell access 
cannot be distinguished anymore. A microfunnel with perfectly smooth surface formed instead. This funnel structure differs in shape and size from the microfabricated funnel described in the previous section. Its diameter at the surface and depth is about a factor of 3 smaller. Microstructures, like those shown in Fig. 5a, b, are expected to be very suitable for patchclamp experiments.

Figure 5c shows a cross section through a microfunnel similar to the one shown in Fig. $5 \mathrm{~b}$ (reflow at $1,100^{\circ} \mathrm{C}$, PSG thickness $1.5 \mu \mathrm{m}$ ). A constriction with a diameter of $0.9 \mu \mathrm{m}$ appeared in the upper part of the tube. The nozzle is buried in the PSG layer after reflow. Smoothing of angles and the surface could be clearly observed. The fact that the constriction is limited to the upper part of the oxide tube is a very interesting feature for the present application. Such small apertures are required to reduce the cell membrane patch size. Smaller aperture openings increase the chance of high seal resistances and reduce the risk of damaging the cell during patch disruption for wholecell recording. On the other hand, the diameter of the rest of the tube is not reduced (about $3 \mu \mathrm{m}$ ). Such large oxide tube diameters provide low-resistant fluidic and electrical access to the cell.

\section{Electrical characterization}

\subsection{Fluidic integration and open resistance measurements}

Before discussing in detail the electrical measurements with the new microchips, their fluidic integration will be briefly described. Figure 1 shows a schematic view of the patch-clamp system. The Si chip with the microaperture is mounted in between two Teflon ${ }^{\mathrm{TM}}$ or plastic plates with PDMS gaskets. Small liquid reservoirs of filtered phosphate-buffer saline (PBS) solution on both sides of the Si chip ( $\approx 1$ and $10 \mu \mathrm{l}$, respectively) immerse the microstructure in the physiological solution. Cells are introduced in the top plate, which incorporates either a small reservoir or channel structures for fluidic exchange and hydrodynamic cell positioning. When a cell is within about $100 \mu \mathrm{m}$ from the aperture, gentle suction through the aperture from the backside of the chip allows positioning and immobilization of the chosen cell. Ideally, a cell should not be in contact with any other surface of the system prior to immobilization to keep the cell membrane perfectly clean and undamaged. Furthermore, any contamination or cell fragments in the solution may stick to the micro-aperture and hinder the formation of a gigaseal. For these reasons, special attention was paid to simplify the fluidic integration and to use easily cleanable components.

Chlorided $\mathrm{Ag}$ wires are integrated on both sides of the chip for the electrical measurements. Typically, a voltage test pulse of $\pm 10 \mathrm{mV}$ is applied to the chip and the current response is recorded. A suitable patchclamp amplifier with a D/A interface (HEKA and pulse software) is used for that purpose.

For an open microfunnel (microstructure shown in Fig. 4c) filled with PBS, i.e. without immobilized cell, a resistance of $1.8 \pm 0.3 \mathrm{M} \Omega$ is found. For modified nozzle-type structures (shown in Fig. 5a), the open resistance is $1.0 \pm 0.1 \mathrm{M} \Omega$. These values determine the access resistance to the cell. They are comparable with typical pipette resistances and reasonably low for patch-clamp experiments. The parallel capacitance is mainly determined by the area of the chip in contact with the saline solution. It is estimated to about $15 \mathrm{pF} /$ $\mathrm{mm}^{2}$ for these chips. With the actual fluidic integration design, this area is about $2 \mathrm{~mm}^{2}$.

\subsection{Seal resistance measurements}

First experiments with the new microstructures described in this paper demonstrate that gigaseals can be obtained. Wild-type Chinese hamster ovary cells ( $\mathrm{CHO}$, average diameter $15 \mu \mathrm{m}$ ) have been used, i.e. unmodified cells that express their endogenous population of ion channels. We use a non-adherent cell line, i.e. no trypsin treatment is required to harvest the cells from the culture dish. They have been removed from the growth medium 2-3 days after splitting the cell culture. The cells are resuspended and washed two times in PBS solution prior to the experiment. At this stage of the development, we focus on a first evaluation of the seal resistance between the cell and the microstructure. Therefore, no specific intra- or extra-cellular solution was used, and the whole microsystem was filled with PBS solution.

Figure 6 shows the time dependence of the current curve after immobilization of a cell and the formation of a gigaseal. A microfunnel as shown in Fig. 4c was used for this experiment (PSG reflow at $900^{\circ} \mathrm{C}$ ). For a voltage test pulse of $\pm 10 \mathrm{mV}$, the corresponding current response $\Delta I$ is $7.5 \mathrm{pA}$, resulting in a seal resistance of $2.6 \mathrm{G} \Omega$. This measurement shows the maximum seal resistance obtained with this structure.

Likewise, the seal formation with $\mathrm{CHO}$ cells has been tested with the improved nozzle-type structure shown in Fig. 5a (PSG reflow at $900^{\circ} \mathrm{C}$ ). Gigaseals have also been obtained with this type of microstructure as shown in Fig. 7. A resistance of $1 \mathrm{G} \Omega$ was found in this 


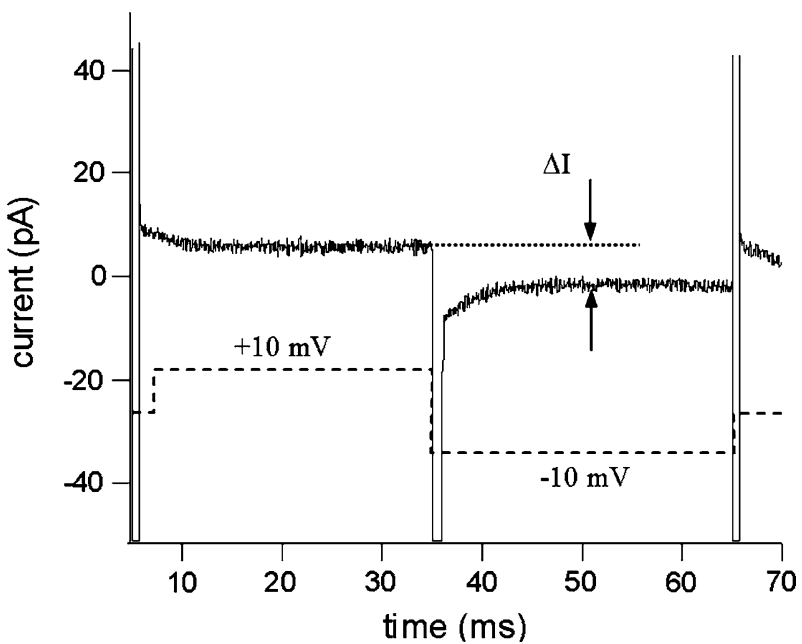

Fig. 6 Electrical characterization of a $\mathrm{CHO}$ cell immobilized and sealed on a microfunnel of the type shown in Fig. 4c. The current response $\Delta I$ to a voltage pulse of $\pm 10 \mathrm{mV} / 30 \mathrm{~ms}$ (dashed line) is measured (averaged ten times). $\Delta I$ is about $7.5 \mathrm{pA}$, indicating a seal resistance $R \approx 2.6 \pm 0.2 \mathrm{G} \Omega$

case with a current response $\Delta I \approx 20 \mathrm{pA}$ (solid line) to a voltage test pulse $( \pm 10 \mathrm{mV} / 10 \mathrm{~ms}$, dashed line $)$. This seal was stable for at least $20 \mathrm{~min}$. After ejecting the cell from the aperture by slight overpressure, the initial open structure resistance was recovered.

In a representative series of ten measurements that were carried out in good experimental conditions, four gigaseals with resistance values above $1 \mathrm{G} \Omega$ had been obtained. For the other recordings the seal resistance varied between 50 and $230 \mathrm{M} \Omega$. In some cases, the chips have been stored for several days after thermal reflow. This result demonstrates the promising potential of the new microstructures. The experiments allowed to observe the dynamics of the gigaseal formation. This will be discussed in more detail below.

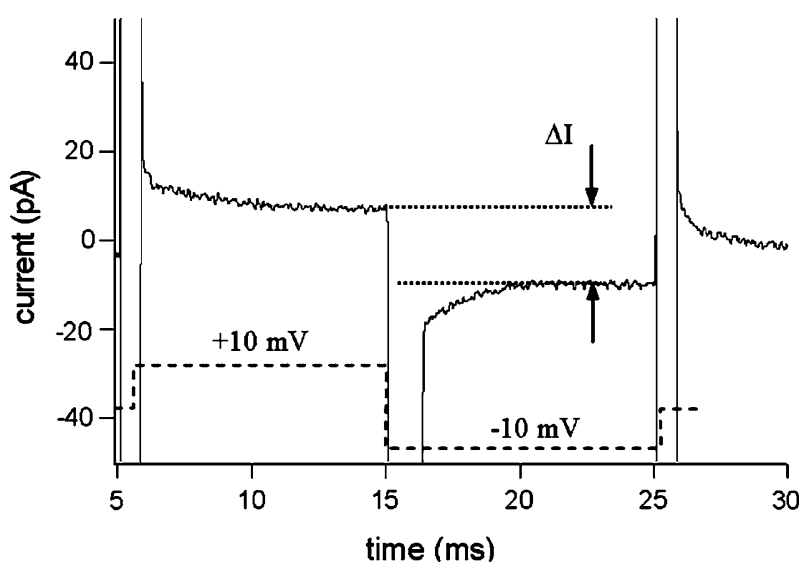

Fig. 7 Seal evaluation of a $\mathrm{CHO}$ cell immobilized on a micronozzle with very smooth surface (as shown in Fig. 5a). The measurement (averaged 20 times) shows a gigaseal with a seal resistance $R \approx 1 \mathrm{G} \Omega$

\section{Discussion}

There is no detailed understanding of the particular physical or biochemical mechanism of the formation of a gigaseal. Our approach to new micro-apertures is to reproduce on-chip an environment very similar to that of a cell being sucked to a pipette tip. Immobilized suspended cells fit easily to these 3-dimensional microstructures and seal with comparatively large contact area. The shape of the coated nozzle shown in Fig. 5a corresponds well with the rounded shape of a firepolished pipette tip. The application of PSG reflow to micro-apertures at high temperatures can be compared to fire polishing, in terms of surface smoothness and surface activation. Surface activation through thermal annealing occurs when strained $\mathrm{Si}-\mathrm{O}$ bonds break up in contact with moisture and form hydroxyl groups at the tip surface that interact with polar phospholipid head groups, via hydrogen bonds or direct electrostatic interaction. Also the relatively high ion content of this doped glass layer may trigger physicochemical interactions similar to fire-polished borosilicate glass.

Two phases can be clearly distinguished during seal formation of a $\mathrm{CHO}$ cell with the here-presented microstructures. As soon as a well-shaped cell is immobilized on the micro-aperture, a slight negative pressure gradient is applied ( $\Delta p$ up to about -30 mbar). By this, it should be possible to increase the resistance to values between 100 and $200 \mathrm{M} \Omega$ within a short period of time (i.e. less than about $30 \mathrm{~s}$ ). During this first phase, the strength of suction is directly correlated with the increase of the seal resistance. Fine-tuning of the pressure gradient is often necessary to ensure that the resistance keeps rising continuously. Suction through a mouthpiece is very suitable for this purpose. The resistance value at this point is not related to a specific seal interaction between the cell membrane and the chip aperture but rather reflects the quality of both surfaces and thus the degree of how close they can be "mechanically" brought together. A good precondition for gigaseal formation is given when only very light suction has to be applied, just enough to fit the cell to the 3-dimensional shape of the microstructure.

However, in practice, if the resistance stagnates below $100 \mathrm{M} \Omega$, the formation of a gigaseal cannot be expected, even if the negative pressure gradient is strongly increased. The increase of the seal resistance behaves clearly different with the new smooth microstructures compared to microstructures with relatively rough surface, such as the previously described bare $\mathrm{SiO}_{2}$ micronozzles. In the latter case, only a small resistance increase can be obtained by applying relatively strong suction (Lehnert et al. 2002). Under these 
conditions, part of the cell is pulled into the oxide tube under the micro-aperture. The cell is strongly deformed, plugs the microtube and may even be sucked through. The problem of surface roughness introduced by dry etching could be solved by the present PSG reflow technique. With the pressure gradients required for the smooth microstructures, the plastic deformation of the cell membrane is much less (this can be seen after repulsing the cell from the micro-aperture).

The second phase of the gigaseal formation starts once a good contact between cell and aperture has been established, as described above. From then on, no more external manipulation is required, and the applied pressure can be kept constant. The resistance increases rapidly (within a few seconds) and continuously by itself to the giga-ohm range, i.e. up to about $1-$ $2 \mathrm{G} \Omega$ for our devices. Thereafter, the seal resistance remains constant, even when the external negative pressure gradient is released. The observed dynamics of the seal formation is very similar to conventional glass pipettes.

Seal formation on planar pores fabricated by $\mathrm{SiO}_{2}$ CVD deposition on $\mathrm{Si}$ apertures was investigated by other authors for different diameters (Pantoja et al. 2004). Gigaseals have been obtained with micropores, but surface roughness and shape were mentioned as limiting factors for high seal resistance formation on nanopores. Our method may present a solution for this problem, as extremely smooth sub-micron apertures can be readily fabricated.

\section{Conclusions}

Phosphosilicate glass layer deposition and reflow applied to microstructures is a promising new technique for microdevices requiring very smooth surface and rounded shape that can otherwise not be fabricated with conventional micromachining processes. Such microstructures are well suited for cell-based bio-microsystems, where stable immobilization of living cells is necessary, while minimizing the risk of damaging the cell membrane. For the present patch-clamp microsystem this new method brought significant improvement compared to previously studied micro-apertures. Cells fit perfectly to this new type of PSG-coated microfunnels or micronozzles and the formation of gigaseals could be studied.

Acknowledgments The authors would like to thank Prof. J.-D. Horisberger (Department of Pharmacology and Toxicology, UNIL, CH-Lausanne) for stimulating discussions on issues related to the patch-clamp technique. The authors also thank the EPFL Center of MicroNano Technology (EPFL-CMI), in particular Dr. C. Hibert (dry etching) and Dr. Ph. Langlet (PSG deposition) for help in the process development, as well as Dr. D. Bouvet (EPFL-LEG1, chemical-mechanical polishing) and $\mathrm{J}$. Steen (EPFL-LMIS1, AFM measurements).

\section{References}

Adams AC, Capio CD (1981) Planarization of phosphorusdoped silicon dioxide. J Electrochem Soc 128:423-429

Ashcroft FM (2000) Ion chanels and disease: channelopathies. Academic, San Diego

Asmild M et al (2003) Upscaling and automation of electrophysiology: toward high throughput screening in ion channel drug discovery. Receptors Channels 9:49-58

Bennett PB, Guthrie HRE (2003) Trends in ion channel drug discovery: advances in screening technologies. Trends Biotechnol 21:563-569

Bruggemann A et al (2003) High quality ion channel analysis on a chip with the NPC (c) technology. Assay Drug Dev Technol 1:665-673

Comley J (2003) Patchers versus screeners-divergent opinion on high throughput electro-physiology. Drug Discovery World: (Fall) 47-57

Hamill OP et al (1981) Improved patch-clamp techniques for high-resolution current recording from cells and cell-free membrane patches. Pflugers Archiv 391:85-100

Hara T, Suzuki H, Furukawa M (1984) Reflow of PSG layers by halogen lamp short duration heating technique. Jpn J Appl Phys Part 2 Lett 23:L452-L454

Ionescu-Zanetti C et al (2005) Mammalian electrophysiology on a microfluidic platform. Proc Natl Acad Sci USA 102:91129117

Jansen H et al (1995) The black silicon method-a universal method for determining the parameter setting of a fluorinebased reactive ion etcher in deep silicon trench etching with profile control. J Micromech Microeng 5:115-120

Klemic KG et al (2002) Micromolded PDMS planar electrode allows patch clamp electrical recordings from cells. Biosens Bioelectron 17:597-604

Klemic KG, Klemic JF, Sigworth FJ (2005) An air-molding technique for fabricating PDMS planar patch-clamp electrodes. Pflugers Archiv 449:564-572

Lehnert T, Gijs M (2004) Patch-clamp microsystems. In: Andersson $\mathrm{H}$, van de Berg A (eds) Lab on chips for cellomics. Kluwer, Dordrecht

Lehnert $\mathrm{T}$ et al (2002) Realization of hollow $\mathrm{SiO} 2$ micronozzles for electrical measurements on living cells. Appl Phys Lett 81:5063-5065

Lehnert T, Laine A, Gijs M (2003) Surface modification of $\mathrm{SiO}_{2}$ micro-nozzles for patch-clamp measurements on-chip. In: Proceedings of 7 th international conference on micro total analysis systems, Squaw Valley, California, USA, Transducers Research Foundation

Luginbuhl $\mathrm{P}$ et al (2000) Femtoliter injector for DNA mass spectrometry. Sens Actuators B Chem 63:167-177

Netzer R et al (2001) Screening lead compounds for QT interval prolongation. Drug Discov Today 6:78-84

Pantoja R et al (2004) Silicon chip-based patch-clamp electrodes integrated with PDMS microfluidics. Biosens Bioelectron 20:509-517

Sakmann B, Neher E (1995) Single-channel recording. Plenum Press, New York 
Schroeder K et al (2003) IonWorks (TM) HT: a new highthroughput electrophysiology measurement platform. J Biomol Screen 8:50-64

Sigworth FJ, Klemic KG (2005) Microchip technology in ionchannel research. IEEE Trans Nanobioscience 4:121-127

van Stiphout P et al (2005) 3D microfluidic chip for automated patch-clamping VDE Verband der Elektrotechnik Elektronik Informationstechnik e.V., Berlin

Vassiliev VY et al (2000) Properties and gap-fill capability of HPD-CVD phosphosilicate glass films for subquartermicrometer ULSI device technology. Electrochem Solid State Lett 3:80-83

Wang XB, Li M (2003) Automated electrophysiology: high throughput of art. Assay Drug Dev Technol 1:695-708
Willumsen NJ et al (2003) High throughput electrophysiology: new perspectives for ion channel drug discovery. Receptors Channels 9:3-12

Wood C, Williams C, Waldron GJ (2004) Patch clamping by numbers. Drug Discov Today 9:434-441

Worley JF, Main MJ (2002) An industrial perspective on utilizing functional ion channel assays for high throughput screening. Receptors Channels 8:269-282

$\mathrm{Xu} \mathrm{J}$ et al (2001) Ion-channel assay technologies: quo vadis? Drug Discov Today 6:1278-1287

$\mathrm{Xu} \mathrm{J}$ et al (2003) A benchmark study with SealChip (TM) planar patch-clamp technology. Assay Drug Dev Technol 1:675684 